\title{
TRATAMIENTO QUIRÚRGICO DE LOS TUMORES DE PARÓTIDA: EXPERIENCIA DE 10 AÑ OS*
}

\author{
Drs. Ricardo Yáñez M. ${ }^{1}$, Francisco Javier Loyola B. ${ }^{1,2}$, Int. Cristóbal Maíz H. ${ }^{3}$, \\ Drs. Pablo Mariangel P. ${ }^{2}$, Jorge Cornejo F. ${ }^{1}$, Raúl Martínez R. ${ }^{1,2}$, Mariana Valenzuela G. ${ }^{1}$
}

1 Cirugía de Cabeza y Cuello. Servicio de Cirugía. Hospital Dr. Sótero del Río.

2 Facultad de Medicina Pontificia Universidad Católica de Chile.

3 Interno de Medicina, Pontificia Universidad Católica de Chile.

Santiago, Chile.

\section{Abstract}

\section{Surgical treatment of parotid neoplasms: A 10 years experience}

Background: Parotid neoplasms correspond to 3\% of head and neck tumors. Most are benign, treatment is parotidectomy. Aims: To analyze the results of treatment, histology, complications and recurrence. Patients and Methods: Retrospective analysis of patients with parotid tumors treated in our center among 2001-2010. Results: The series consisted of 82 patients, 52 (63\%) women. Average age: 46 years (range, 17-77), 73 $(89 \%)$ had benign tumors, 7 (8.5\%) were malignant and $2(2.4 \%)$ had chronic inflammatory disease. The most common benign tumors were pleomorphic adenoma (55\%) and Warthin's tumor (20\%). The most common malignant tumors were mucoepidermoid carcinoma (5\%) and acinar cell carcinoma (4\%). The technique was total parotidectomy in 10 patients $(12 \%)$, total suprafacial $53(64.6 \%)$ and partial in $19(23 \%)$. The most common complication was transient facial nerve dysfunction in the immediate postoperative period in 38 cases (46.3\%), $14(17 \%)$ had surgical bed depression, 15 (18.3\%), dysesthesia periauricular, $2(2.4 \%)$ permanent paralysis of the facial nerve (tumor) and $6(7.3 \%)$ Frey Syndrome. No patient had a disagreement with his scar and pain periauricular, there were no recurrences. There was lesser transient facial nerve paralysis with partial suprafacial parotidectomy than with total suprafacial parotidectomy (21\% and 53\%), RR 0.4 IC 95\% (0.16-0.99). Discussion: The application of less invasive surgical techniques such as partial parotidectomy suprafacial allow comparable results in benign conditions, with low morbidity without increasing recurrence.

Key words: Parotid neoplasm, head and neck neoplasms, Warthin tumor, salivary gland diseases.

\section{Resumen}

Introducción: Las neoplasias parotídeas corresponden al 3\% de los tumores de cabeza y cuello. La mayoría son benignas, el tratamiento es la parotidectomía. Objetivo: Analizar los resultados del tratamiento, histología, complicaciones y recurrencia. Pacientes y Métodos: Análisis retrospectivo de los pacientes con tumores parotídeas intervenidos en nuestro centro entre 2001-2010. Resultados: La serie estuvo compuesta

\footnotetext{
Los autores no refieren conflictos de interés.

Correspondencia: Dr. Ricardo Yáñez M. yanezmartinez@gmail.com
}

*Recibido el 1 de julio de 2013 y aceptado para publicación el 11 de noviembre de 2013. 
por 82 pacientes, 52 (63\%) mujeres. La edad promedio: 46 años (rango, 17-77); 73 (89\%) tuvieron tumores benignos, $7(8,5 \%)$ malignos y $2(2,4 \%)$ presentaron enfermedad inflamatoria crónica. Los tumores benignos más frecuentes fueron el adenoma pleomorfo (55\%) y el tumor de Warthin $(20 \%)$. Los tumores malignos más frecuentes fueron el carcinoma mucoepidermoide (5\%) y el carcinoma de células acinares (4\%). La técnica fue parotidectomía total en 10 pacientes (12\%), suprafacial total en $53(64,6 \%)$ y parcial en 19 (23\%). La complicación más frecuente fue la disfunción transitoria del nervio facial en el postoperatorio inmediato en 38 casos $(46,3 \%) ; 14(17 \%)$ presentaron depresión del lecho operatorio, 15 (18,3\%) disestesia periauricular, $2(2,4 \%)$ parálisis permanente del nervio facial (tumor maligno) y 6 (7,3\%) Síndrome de Frey. Ningún paciente presentó disconformidad con su cicatriz ni dolor periauricular; no hubo recidivas. Se observó menos parálisis transitoria del nervio facial con la parotidectomía suprafacial parcial que con la parotidectomía suprafacial total (21\% y 53\%, respectivamente), RR 0,4 IC 95\% (0,16-0,99). Discusión: La aplicación de técnicas quirúrgicas menos agresivas como la parotidectomía suprafacial parcial permite obtener resultados comparables en patologías benignas, con bajas tasas de morbilidad, sin aumentar la recurrencia.

Palabras clave: Parótida, adenoma pleomorfo, tumor de Warthin, nervio facial.

\section{Introducción}

Los tumores de parótida corresponden al $80 \%$ de los tumores de glándulas salivales y al $3 \%$ de todos los tumores de cabeza y cuello ${ }^{1,2}$. De ellos, un $80 \%$ son benignos y se presentan generalmente como un aumento de volumen indoloro, salvo algunos casos de malignidad ${ }^{2,3}$. El tratamiento de elección es la parotidectomía ${ }^{4-6}$. La técnica quirúrgica ha cambiado con el paso del tiempo entre sus formas más radicales y conservadoras, buscando el equilibrio entre la resección completa de la masa tumoral, con el consiguiente menor riesgo de recidiva, y por otro lado, la necesidad de disminuir las complicaciones postoperatorias asociadas. De esta forma, la cirugía parotídea se inicia a principios de 1800 con la enucleación de las masas tumorales, presentando baja tasa de complicaciones postoperatorias pero una alta recidiva, explicada principalmente por la permanencia de la cápsula tumoral in situ del tumor más frecuente, el adenoma pleomorfo, de naturaleza benigna ${ }^{7}$. Este hecho dio paso a cirugías más radicales como la parotidectomía total y suprafacial, logrando disminuir la recidiva tumoral, pero al mismo tiempo aumentando las complicaciones como la parálisis del nervio facial, el síndrome de Frey y las alteraciones cosméticas. En la actualidad la parotidectomía suprafacial parcial y la disección extracapsular se han posicionado con fuerza, tras demostrar su efectividad en la disminución de las mencionadas complicaciones, sin implicar un aumento de la tasa de recidiva $^{8}$. En este estudio analizamos los resultados del tratamiento quirúrgico de los tumores parotídeos en nuestro centro.

\section{Pacientes y Métodos}

Análisis retrospectivo de los 82 pacientes sometidos a parotidectomía en nuestro hospital entre los años 2001 y 2010. Se incluyó casos tanto de tumores benignos como malignos. Se realizó parotidectomías totales, parotidectomías suprafaciales y parotidectomías suprafaciales parciales, dependiendo del caso de cada paciente. Todos los pacientes fueron sometidos a ecografía de partes blandas previo a la cirugía para evaluar la naturaleza del tumor. En los pacientes con sospecha de malignidad se realizó citología mediante punción por aguja fina. Todos los pacientes con tumores malignos fueron etapificados según la clasificación TNM del American Joint Comittee on Cancer. La técnicas quirúrgicas utilizadas descritas fueron: a) parotidectomía total: disección completa del nervio facial y remoción de toda la glándula; b) parotidectomía suprafacial: disección completa del nervio facial y remoción del tejido parotídeo superficial al nervio; y c) parotidectomía suprafacial parcial: resección selectiva del tumor con margen de tejido normal ${ }^{9}$. Las complicaciones analizadas fueron parálisis del nervio facial, transitoria y permanente, depresión del lecho operatorio, disestesia periauricular, síndrome de Frey, disconformidad con la cicatriz, discomfort o dolor periauricular y recidiva tumoral. La información sobre tipo de cirugía realizada, histología, complicaciones y recurrencia fue obtenida a partir de la revisión de las historias clínicas, controles clínicos seriados y aplicación de encuesta al paciente en el postoperatorio tardío (mínimo un año posterior a la intervención). Para el análisis estadístico se utilizó el riesgo relativo, reducción de riesgo relativo y el intervalo de confianza correspondiente.

\section{Resultados}

Entre los años 2001 y 2010 un total de 82 pacientes se sometió a parotidectomía en nuestro centro, de los cuales 52 (63\%) fueron mujeres. El promedio de edad de este grupo fue de 46 años (rango, 17-77 
años). De los tumores operados, 73 (89\%) de ellos resultaron ser benignos, $7(8,5 \%)$ malignos, y 2 $(2,4 \%)$ presentaron enfermedad inflamatoria crónica. En cuanto a la histología, la más frecuente entre los tumores benignos resultó ser el adenoma pleomórfico, en un $55 \%$ de los casos, seguido del tumor de Warthin o cistoadenoma papilar linfomatoso en un $20 \%$ de los casos. Entre los tumores malignos el más frecuente fue el carcinoma mucoepidermoide, en cuatro pacientes, y luego el carcinoma de células acinares, en tres pacientes. En el grupo de pacientes con tumores malignos uno estaba en estadío II, uno en estadío III y los cinco pacientes restantes en estadio IV. A todos se les realizó una parotidectomía total; además se les realizó una disección cervical radical modificada a aquellos que presentaban adenopatías. Todos los pacientes en estadío III y IV fueron sometidos a radioterapia posterior a la cirugía. Los resultados de histología tumoral se presentan en la Tabla 1.

La técnica quirúrgica utilizada fue parotidectomía total en 10 pacientes (12\%), suprafacial en 53 pacientes $(64,6 \%)$ y suprafacial parcial en 19 pacientes $(23 \%)$. La complicación más frecuente fue la parálisis transitoria del nervio facial, en el período postoperatorio inmediato, en 38 pacientes $(46,3 \%)$; 14 pacientes $(17 \%)$ presentaron depresión del lecho operatorio, 15 pacientes $(18,3 \%)$ presentaron disestesia periauricular, dos pacientes $(2,4 \%)$ presentaron parálisis permanente del nervio facial (ambos con tumores malignos de parótida), y seis pacientes (7,3\%) presentaron Síndrome de Frey (evaluado mediante interrogatorio clínico). Ningún paciente quedó disconforme con su cicatriz ni presentó dolor periauricular. Ninguno de los pacientes presentó recidiva tumoral (Tabla 2). Se realizó un análisis de las complicaciones observadas en los pacientes con

Tabla 1. Histología de los tumores parotídeos

\begin{tabular}{|lc|}
\hline Histología & n pacientes, (\%) \\
Tumores benignos & $73(89 \%)$ \\
Adenoma pleomorfo & $45(55 \%)$ \\
Tumor de Warthin & $16(20 \%)$ \\
Oncocitoma & $5(6 \%)$ \\
Lesión linfoepitelial benigna & $4(5 \%)$ \\
Adenoma monomórfico & $3(4 \%)$ \\
Tumores malignos & $7(9 \%)$ \\
Carcinoma mucoepidermoide & $4(5 \%)$ \\
Carcinoma de células acinares & $3(4 \%)$ \\
Enfermedad inflamatoria crónica & $2(2 \%)$ \\
Total & 82 pacientes \\
\hline
\end{tabular}

patología benigna sometidos a una parotidectomía, al comparar las técnicas de parotidectomía suprafacial con la parotidectomía suprafacial parcial se observó una reducción significativa de la parálisis transitoria del nervio facial en los pacientes con la técnica parcial (53\% y $21 \%$, respectivamente), con un RR (riesgo relativo) de 0,4 IC 95\% $(0,16-0,99)$ y una RRR (reducción del riesgo relativo) de $60 \%$ IC 95\% (0,16-0,99); al comparar la depresión del lecho quirúrgico, disestesia periaricular, prevalencia de Síndrome de Frey, disconformidad con la cicatriz, dolor o discomfort periauricular y recurrencia tumoral, la diferencia no fue estadísticamente significativa (Tabla 3).

\section{Discusión}

Los tumores benignos de parótida, en nuestra serie resultaron ser algo más frecuentes entre los tumores parotídeos (89\%) que lo publicado en la literatura ${ }^{1-3}$. Aún así, las proporciones de sus diferentes tipos histológicos son concordantes con los resultados de otros estudios. El tumor benigno más frecuente en nuestra serie fue el adenoma pleomorfo (55\%), del cual se ha determinado que corresponde entre el 60,6 y $76,2 \%$ de los tumores de parótida, se presenta en general entre la segunda y cuarta décadas de la vida y es más común en mujeres (hombre:mujer 1:1,4); asimismo encontramos el tumor de Warthin en un $20 \%$ de los casos, cuya prevalencia se ha estimado entre un 5-20\% de los tumores parotídeos; además se encontró un $2 \%$ de incidencia de enfermedad inflamatoria crónica, referida usualmente en otras publicaciones ${ }^{4}$. Dada la alta prevalencia de lesiones benignas entre los tumores de parótida, es esperable que cirugías menos

Tabla 2. Complicaciones en todos los pacientes sometidos a parotidectomía por tumores benignos, malignos y patologías inflamatorias

\begin{tabular}{|lc|}
\hline Complicación & n pacientes, \% \\
\hline Parálisis transitoria nervio facial & $38(46,3 \%)$ \\
\hline Parálisis permanente del nervio facial* & $2(2,4 \%)$ \\
Depresión del lecho operatorio & $14(17 \%)$ \\
\hline Disestesia periauricular & $15(18,29 \%)$ \\
Síndrome de Frey & $6(7,3 \%)$ \\
Disconformidad con la cicatriz & 0 \\
Dolor periauricular & 0 \\
Recidiva tumoral & 0 \\
\hline
\end{tabular}

*Ambos pacientes con tumor maligno de parótida. 
Tabla 3. Complicaciones postoperatorias según técnica quirúrgica en pacientes con patología benigna

\begin{tabular}{|c|c|c|c|c|c|}
\hline & $n=75$ & $\begin{array}{c}\text { Parotidectomía } \\
\text { total } \\
(\mathbf{n}=\mathbf{3})\end{array}$ & $\begin{array}{l}\text { Parotidectomía } \\
\text { suprafacial } \\
(\mathbf{n}=\mathbf{5 3})\end{array}$ & $\begin{array}{c}\text { Parotidectomía } \\
\text { suprafacial parcial } \\
(\mathbf{n}=\mathbf{1 9 )}\end{array}$ & $\begin{array}{l}\text { Parotidectomía supra- } \\
\text { facial vs parotidectomía } \\
\text { suprafacial parcial }\end{array}$ \\
\hline $\begin{array}{l}\text { Parálisis transitoria } \\
\text { nervio facial }\end{array}$ & $\begin{array}{c}35 \\
(46,7 \%)\end{array}$ & $\begin{array}{c}3 \\
(100 \%)\end{array}$ & $\begin{array}{c}28 \\
(53 \%)\end{array}$ & $\begin{array}{c}4 \\
(21 \%)\end{array}$ & $\begin{array}{c}\text { RR } 0,4 \\
\text { IC 95\% }(0,16-0,99) \\
\text { RRR } 60 \% \\
\text { IC 95\% }(0,16-0,99)\end{array}$ \\
\hline $\begin{array}{l}\text { Depresión del lecho } \\
\text { quirúrgico }\end{array}$ & $\begin{array}{c}12 \\
(16 \%)\end{array}$ & $\begin{array}{c}3 \\
(100 \%)\end{array}$ & $\begin{array}{c}9 \\
(17 \%)\end{array}$ & 0 & $\begin{array}{c}* \\
\text { RR } 0,14 \\
\text { IC } 95 \%(0,01-2,33) \\
\text { RRR } 86 \% \\
\text { IC } 95 \%(0,01-2,33)\end{array}$ \\
\hline $\begin{array}{l}\text { Disestesia } \\
\text { periauricular }\end{array}$ & $\begin{array}{c}14 \\
(18,7 \%)\end{array}$ & $\begin{array}{c}2 \\
(66,7 \%)\end{array}$ & $\begin{array}{c}10 \\
(18,8 \%)\end{array}$ & $\begin{array}{c}2 \\
(10,5 \%)\end{array}$ & $\begin{array}{c}* \\
\text { RR } 0,56 \\
\text { IC } 95 \%(0,13-2,32) \\
\text { RRR } 44 \% \\
\text { IC } 95 \%(0,13-2,32)\end{array}$ \\
\hline Síndrome de Frey & $\begin{array}{c}3 \\
(4 \%)\end{array}$ & $\begin{array}{c}3 \\
(4 \%)\end{array}$ & 0 & 0 & $*$ \\
\hline $\begin{array}{l}\text { Disconformidad con } \\
\text { la cicatriz }\end{array}$ & 0 & 0 & 0 & 0 & $*$ \\
\hline $\begin{array}{l}\text { Dolor o discomfort } \\
\text { periauricular }\end{array}$ & 0 & 0 & 0 & 0 & $*$ \\
\hline Recurrencia tumoral & 0 & 0 & 0 & 0 & $*$ \\
\hline
\end{tabular}

*no significativo.

invasivas, con buenos márgenes, pudieran resultar igualmente efectivas y menos mórbidas.

Los tumores malignos de parótida $(8,5 \%)$ resultaron ser menos frecuentes en nuestro estudio que lo descrito en la literatura (15-40\%). A pesar de esto, al igual que en la experiencia internacional, el tumor maligno más frecuente fue el carcinoma mucoepidermoide, presente en un $5 \%$ de todas las lesiones, seguido por el carcinoma de células acinares (4\%). Respecto a ello, es importante mencionar que en el caso de los tumores malignos, la extensión de la lesión es un predictor más importante de los resultados quirúrgicos que su tipo histológico. Entre un 10 a $32 \%$ de ellos se presentan con dolor y suelen debutar como una masa adherida a planos profundos y/o a la piel. Otros predictores de malignidad de los tumores de parótida son la paresia preoperatoria del nervio facial (observada en 9-25\% de los casos) y la presencia de adenopatía cervical ipsilateral (en 13$25 \%$ de los casos $)^{6,7}$. Las imágenes como tomografía axial computada (TC) y resonancia magnética (RM) nos permiten evaluar la profundidad y en el caso de la RM la relación del tumor con el nervio facial. Por otro lado, la recurrencia locorregional se puede disminuir utilizando radioterapia adyuvante post qui- rúrgica, indicada cuando existe enfermedad en etapa avanzada (III-IV), dudas acerca de la seguridad de los márgenes o hallazgo histológico ominoso como tumor de alto grado, invasión neural o vascular ${ }^{8,9}$.

Entre las complicaciones post parotidectomía se encuentran la parálisis del nervio facial, tanto transitoria como permanente, el síndrome de Frey, las alteraciones estéticas derivadas de una cicatriz en territorio facial y de la posibilidad de depresión del lecho operatorio, los hematomas, seromas y neuromas. La incidencia del síndrome de Frey o sudoración gustativa post parotidectomía varía ampliamente en la literatura, debido fundamentalmente al tipo de cirugía, el diseño de los estudios, el tiempo de seguimiento y las formas de evaluación del mismo. De esta forma se ha reportado que post parotidectomía sólo un $10 \%$ de los pacientes manifiesta espontáneamente síntomas compatibles con la enfermedad, un $30-50 \%$ si se les pregunta, y hasta un $96 \%$ tiene un test de almidón yodado positivo, el cual se ha validado para hacer el diagnóstico de síndrome de Frey ${ }^{10}$. Se ha demostrado además, la eficacia de los injertos y colgajos en la prevención de síndrome de Frey post parotidectomía. El injerto de matriz de dermis acelular disminuye el riesgo entre un $82 \%$ 
y $90 \%$ (dependiendo de cómo se evalúe), mientras que el colgajo de músculo esternocleidomastoídeo disminuye un $81 \%$ el riesgo de desarrollar síndrome de Frey ${ }^{11}$. En nuestro estudio obtuvimos una baja tasa de dicha complicación $(7,3 \%)$, todos los casos en parotidectomías totales, lo cual atribuimos a la forma de evaluación, mediante una encuesta, y a la alta cantidad de parotidectomías suprafaciales parciales realizadas.

Una de las complicaciones más temidas de las parotidectomías es la parálisis del nervio facial, ya sea transitoria o permanente. Para evitarla, se utilizan puntos de referencia que permiten identificar el tronco y luego seguir el recorrido de dicho nervio. Algunos equipos además monitorean su función durante la cirugía. A pesar de estos esfuerzos, se ha comprobado que la lesión del nervio facial es directamente proporcional al tamaño tumoral y que en la misma línea, una cirugía más extensa conlleva un mayor riesgo de parálisis transitoria del mismo. Por lo mismo la incidencia reportada varía notablemente según el tipo de cirugía. Lin CC et al, en un estudio retrospectivo de 271 pacientes, reporta una tasa de parálisis transitoria de $18 \%$ y una tasa de parálisis permanente de $3 \%$, con diferentes técnicas quirúrgicas, $88 \%$ de estas últimas en relación a lesiones malignas que por lo general implican una cirugía mayor. Eng CY et al, reporta una tasa de $56 \%$ y $3,6 \%$ de parálisis transitoria y permanente del nervio facial respectivamente, sin diferencias significativas entre equipos quirúrgicos de diferentes especialidades ${ }^{12}$. Una revisión sistemática realizada por Umapathy $\mathrm{N}$ et al, muestra que la parálisis transitoria del nervio facial ocurre entre un 8,8 y $76 \%$ de los pacientes, mientras que la parálisis permanente se observa en un 0 a $14 \%$ de ellos ${ }^{13}$. En nuestra experiencia la parálisis transitoria del nervio facial se presentó en un $46,3 \%$ de los pacientes y la parálisis permanente en $2,4 \%$, lo cual es concordante la experiencia internacional descrita. Cabe mencionar que los 2 pacientes con una parálisis permanente del nervio facial corresponden a pacientes que presentaban lesiones malignas.

En cuanto a las diferencias según técnica quirúrgica, varios estudios demuestran que aquellas formas más acotadas, como son la disección extracapsular y la parotidectomía suprafacial parcial, tienen considerablemente menor tasa de complicaciones que la parotidectomía suprafacial y tota ${ }^{14,15}$. En este estudio observamos una reducción significativa de la parálisis transitoria del nervio facial con parotidectomía suprafacial parcial por sobre la parotidectomía suprafacial total $(21 \%$ y $53 \%$, respectivamente), RR 0,4 IC $95 \%(0,16-0,99)$. En relación a la disección extracapsular, también se ha demostrado su utilidad en la reducción de complica- ciones postoperatorias en múltiples trabajos. Esta es una técnica distinta de la enucleación, pues no deja la cápsula in situ, sino que la reseca con un margen mínimo de 3-4 mm y a diferencia de la parotidectomía suprafacial parcial, no intenta identificar el nervio facial. Está indicada sólo en tumores benignos, por lo que la confirmación de la naturaleza tumoral es fundamental ${ }^{15-17}$.

Respecto al abordaje utilizado para realizar la parotidectomía se ha estudiado que la incisión vía facelift no tiene mayor tasa de complicación y tiene mejores resultados estéticos y menos síndrome de Frey que la incisión de Blair. La evidencia apoya su uso sólo en lesiones benignas. Los tumores anteroinferiores son más difíciles de resecar por esta vía ${ }^{18}$.

Finalmente, al analizar la recidiva de las lesiones operadas en nuestro centro, si bien no se ha visto recidivas, no es posible sacar conclusiones al respecto, debido a que el tiempo de seguimiento no es mayor de 10 años, lo cual es insuficiente para determinar el resultado a largo plazo. Aún con esto, la experiencia observada con la mayoría de los pacientes nos hace ser optimistas.

\section{Conclusiones}

De esta forma, podemos decir que la aplicación de técnicas basadas en la anatomía del nervio facial y cirugías menos agresivas tales como la parotidectomía suprafacial parcial, permiten obtener resultados comparables con técnicas quirúrgicas más extensas a largo plazo y con bajas tasas de morbilidad, sin diferencia en la tasa de recurrencia.

\section{Referencias}

1. Lin CC, Tsai MH, Huang CC, Hua CH, Tseng HC, Huang ST. Parotid tumors: a 10 year experience. Am J Otolaringol. 2008;29:94-100.

2. Smith SL, Komisar A. Limited Parotidectomy: The Role of Extracapsular Dissection in Parotid Gland Neoplasms. Laryngoscope 2007;117:1163-7.

3. Spiro JD, Spiro RH. Cancer of the Parotid Gland: Role of 7th Nerve Preservation. World J Surg. 2003;27:863-7.

4. Kamal SA, Othman EO. Diagnosis and treatment of parotid tumors. J Laryngol Otol. 1997;111:316-21.

5. Parotidectomy. Anatomical considerations. Clin Anat. 2012;25:12-8.

6. Aguirre A, Vent ML. Tumores de la parótida. Tratamiento quirúrgico. Rev Chil Cir. 2004;56:51-6.

7. Valentini V, Fabiani F, Perugini M, Vetrano S, Ianetti G. Surgical Techniques in the treatment of pleomorphic adenoma of the parotid gland: Our experience and review of literature. J Craniofac Surg. 2001;12:565-8. 
8. Johnson JT, Ferlito A, Fagan JJ, Bradley PJ, Rinaldo A. Role of limited parotidectomy in management of pleomorfic adenoma. J Laryngol Otol. 2007;121:1126-8.

9. Tweedie DJ, Jacob A. Surgery of the parotid gland: evolution of techniques, nomenclature and revised classification system. Clin Otolaryngol. 2009;34:303-8.

10. Rustemeyer J, Eufinger H, Bremerich A. The incidence of Frey's syndrome. J Cranioaxillofac Surg. 2008;36:34-7.

11. Li C, Yang X, Pan J, Shi Z, Li L. Graft for prevention of Frey sindrome after parotidectomy: a systematic review and meta-analysis of randomized controlled trials. J Oral Maxillofac Surg. 2013;71:419-27.

12. Eng CY, Evans AS, Quraishi MS, Harkness PA. A comparison of the incidence of facial palsy following parotidectomy performed by ENT and non-ENT surgeons. J Laryngol Otol. 2007;121:40-3.

13. Umapathy N, Holmes R, Basavaraj S, Roux R, Cable HR. Performance of Parotidectomy in nonspecialist cen- ters. Arch Otolaryngol Head Neck Surg. 2003;129:9258.

14. Roh JL, Park CI. Function-Preserving Parotid Surgery for Benign Tumors Involving the Deep Parotid Lobe. J Surg Oncol. 2008;98:42-5.

15. Shehata EA. Extra-capsular dissection for benign parotid tumours. Int J Oral Maxillofac Surg. 2010;39:140-4.

16. Dell'Aversana Orabona $G$, Bonavolontà $P$, Iaconetta $G$, Forte R, Califano L. Surgical management of benign tumors of the parotid gland: extracapsular dissection versus superficial parotidectomy: our experience in 232 cases. J Oral Maxillofac Surg. 2013;71:410-3.

17. Albergotti WG, Nguyen SA, Zenk J, Gillespie MB. Extracapsular dissection for benign parotid tumors: a meta-analysis. Laryngoscope 2012;122:1954-60.

18. Groover N, D'Souza A. Facelift approach for parotidectomy: an evolving aesthetic technique. Otolaryngol Head Neck Surg. 2013;148:548-56. 